\title{
Automatic segmentation of myocardium at risk from contrast enhanced SSFP CMR: validation against expert readers and SPECT
}

\author{
Jane Tufvesson ${ }^{1,2^{*}}$, Marcus Carlsson ${ }^{1}$, Anthony H Aletras ${ }^{1,3}$, Henrik Engblom ${ }^{1}$, Jean-Francois Deux ${ }^{4}$, Sasha Koul ${ }^{5}$, \\ Peder Sörensson ${ }^{6}$, John Pernow ${ }^{6}$, Dan Atar ${ }^{7}$, David Erlinge ${ }^{5}$, Håkan Arheden $^{1}$, Einar Heiberg ${ }^{1,2}$
}

From 19th Annual SCMR Scientific Sessions

Los Angeles, CA, USA. 27-30 January 2016

\section{Background}

Efficacy of reperfusion therapy can be assessed as myocardial salvage index (MSI) by determining the size of myocardium at risk (MaR) and myocardial infarction (MI), (MSI=1-MI/MaR). Cardiovascular magnetic resonance (CMR) can be used to assess MI by late gadolinium enhancement (LGE) and $\mathrm{MaR}$ by either T2-weighted imaging or contrast enhanced SSFP (CESSFP). Automatic segmentation algorithms have been developed and validated for MI by LGE as well as for MaR by T2-weighted imaging. There are, however, no algorithms available for CE-SSFP. Therefore, the aim of this study was to develop and validate automatic segmentation of MaR in CE-SSFP.

\section{Methods}

The automatic algorithm applies surface coil intensity correction and classifies myocardial intensities by Expectation Maximization to define a MaR region based on $a$ priori regional criteria, and infarct region from LGE. Automatic segmentation was validated against manual delineation by expert readers in 183 patients with reperfused acute MI from two multi-center randomized clinical trials (RCT) (CHILL-MI and MITOCARE) and against myocardial perfusion SPECT in an additional set $(\mathrm{n}=16)$. Endocardial and epicardial borders were manually delineated at end-diastole and end-systole. Manual delineation of MaR was used as reference and interobserver variability was assessed for both manual delineation and automatic segmentation of $\mathrm{MaR}$ in a subset of patients $(n=15)$. MaR was expressed as percent of

${ }^{1}$ Dept. of Clinical Physiology, Skåne University Hospital in Lund, Lund University, Lund, Sweden

Full list of author information is available at the end of the article left ventricular mass (\%LVM) and analyzed by bias (mean \pm standard deviation). Regional agreement was analyzed by Dice Similarity Coefficient (DSC) (mean \pm standard deviation).

\section{Results}

MaR assessed by manual and automatic segmentation were $36 \pm 10 \%$ and $37 \pm 11 \%$ LVM respectively with bias $1 \pm 6 \% \mathrm{LVM}$ and regional agreement DSC $0.85 \pm$ $0.08(\mathrm{n}=183)$ (Figure 1). MaR assessed by SPECT and CE-SSFP automatic segmentation were $27 \pm 10 \% \mathrm{LVM}$ and $29 \pm 7 \% \mathrm{LVM}$ respectively with bias $2 \pm 7 \%$ LVM (Figure 1). Inter-observer variability was $0 \pm 3 \%$ LVM for manual delineation and $-1 \pm 2 \% \mathrm{LVM}$ for automatic segmentation.

\section{Conclusions}

Automatic segmentation of MaR in CE-SSFP was validated against manual delineation in multi-center, multivendor studies with low bias and high regional agreement. Bias and variability was similar to inter-observer variability of manual delineation and inter-observer variability was decreased by automatic segmentation. Thus, the proposed automatic segmentation can be used to reduce subjectivity in quantification of MaR in RCT.

\footnotetext{
Authors' details

${ }^{1}$ Dept. of Clinical Physiology, Skåne University Hospital in Lund, Lund University, Lund, Sweden. ${ }^{2}$ Dept. of Biomedical Engineering, Faculty of Engineering, Lund University, Lund, Sweden. ${ }^{3}$ Laboratory of Medical Informatics, School of Medicine, Aristotle University of Thessaloniki, Thessaloniki, Greece. ${ }^{4}$ Dept. of Cardiology, Henri Mondor Hospital, Creteil, France. ${ }^{5}$ Dept. of Cardiology, Lund University, Lund, Sweden. ${ }^{6}$ Dept. of Medicine, Karolinska Institutet, Karolinska University Hospital, Stockholm, Sweden. ${ }^{7}$ Dept. of Cardiology B, Oslo, University Hospital Ullevål and Faculty of Medicine, University of Oslo," Oslo, Norway.
} 


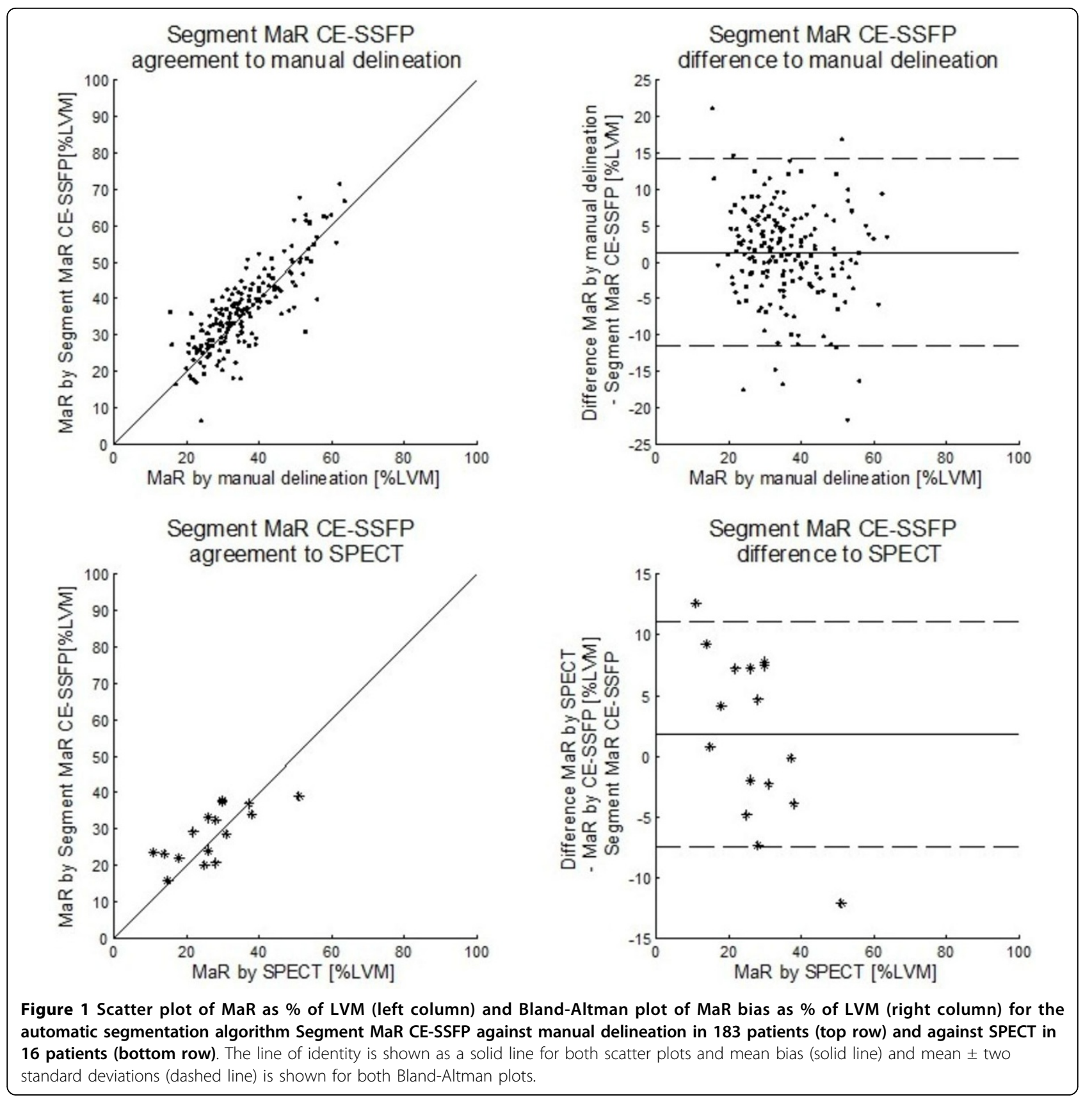

Published: 27 January 2016

doi:10.1186/1532-429X-18-S1-P222

Cite this article as: Tufvesson et al:: Automatic segmentation of myocardium at risk from contrast enhanced SSFP CMR: validation against expert readers and SPECT. Journal of Cardiovascular Magnetic Resonance 2016 18(Suppl 1):P222.
Submit your next manuscript to BioMed Central and take full advantage of:

- Convenient online submission

- Thorough peer review

- No space constraints or color figure charges

- Immediate publication on acceptance

- Inclusion in PubMed, CAS, Scopus and Google Scholar

- Research which is freely available for redistribution 